\title{
¿LOS PRINCIPALES DESTINOS SON TAMBIÉN LAS PRINCIPALES POTENCIAS EN LA INVESTIGACIÓN EN TURISMO? LA EXPERIENCIA ESPAÑOLA
}

\author{
ARE THE MAIN DESTINATIONS THE MAIN PRODUCERS OF \\ TOURISM RESEARCH TOO? \\ THE SPANISH EXPERIENCE
}

\begin{abstract}
Alfonso Vargas Sanchez
Universidad de Huelva, España

Aceptado: 24 de julio de 2014

\section{RESUMEN}

Hoy en día no cabe concebir un modelo de desarrollo turístico que no se alimente de la investigación científica, conforme al denominado por Etzkowitz (2008) modelo de la triple hélice. Es por ello que hemos analizado la relación que pudiera existir entre la importancia del turismo en un país o región y el peso a nivel internacional de su producción científica. Así, hemos podido constatar el crecimiento experimentado por la producción española en lo que va del siglo, que ha llevado a España a situarse, en consonancia con su peso en el turismo mundial, como uno de los países punteros en investigación, aunque aún lejos de la hegemonía de Estados Unidos y Reino Unido. A escala nacional, también ha sido identificada la contribución de las comunidades autónomas y de las universidades a la generación de conocimiento científico a nivel internacional, y se hace un balance de las principales revistas en las que los trabajos han sido publicados.
\end{abstract}

Palabras clave: Investigación, ISI, producción científica, turismo.

\begin{abstract}
It is unconceivable today a model of tourism development without a strong connection to the scientific research community, according to the triple helix model, authored by Etzkowitz (2008). This is why we have analyzed the relationship that might exist between the importance of tourism in a country or region and the weight of its international scientific output. In this sense, we have seen the growth experienced during this century by the Spanish scientific production, which has been placed, in line with its weight in the world tourism, as one of the leading countries in research, although still far from the hegemony of the United States and United Kingdom. Nationally, the contribution of regions and universities to the generation of scientific knowledge at international level has been also identified, as well as major journals in which the papers have been published.
\end{abstract}

Key words: Research, ISI, scientific output, tourism 


\section{Introducción}

¿Existe correlación entre la posición de un país en el ránking mundial del turismo y la producción investigadora en este campo? ¿Son los países que dominan la industria turística los que más conocimiento producen?

Si medimos la primera variable en función del número de llegadas internacionales de turistas, los datos de la Organización Mundial del Turismo (OMT) correspondientes a 2011 nos muestran el panorama que sigue (Tabla1), con los siguientes países líderes:

Tabla 1

\begin{tabular}{lccccc}
\hline País & $\begin{array}{c}\text { Millones de turistas } \\
\text { internacionales } \\
\text { (a) }\end{array}$ & $\begin{array}{c}\text { Posición en el } \\
\text { ránking } \\
\text { (a) }\end{array}$ & $\begin{array}{c}\text { Miles de millones } \\
\text { de US\$ } \\
\text { (a) }\end{array}$ & $\begin{array}{c}\text { Contribución } \\
\text { total al PIB (\%) } \\
\text { (b) }\end{array}$ & $\begin{array}{c}\text { Contribución total } \\
\text { al empleo (\%) } \\
\text { (b) }\end{array}$ \\
\hline Francia & 79,5 & 1 & 53,8 & 9,7 & 10,9 \\
EE.UU. & 62,3 & 2 & 116,3 & 8,6 & 10,1 \\
China & 57,6 & 3 & 48,5 & 9,2 & 8,3 \\
España & 56,7 & 4 & 59,9 & 15,3 & 15,4 \\
Italia & 46,1 & 5 & 43,0 & 10,4 & 11,8 \\
Turquía & 29,3 & 6 & 23,0 & 11,0 & 8,2 \\
Reino Unido & 29,2 & 7 & 35,9 & 6,8 & 7,5 \\
Alemania & 28,4 & 8 & 38,8 & 4,6 & 4,9 \\
Malasia & 24,7 & 9 & 18,3 & 15,0 & 13,1 \\
México & 23,4 & 10 & 11,9 & 12,5 & 13,8 \\
\hline
\end{tabular}

Fuentes: (a) World Tourism Organization; (b) World Travel \& Tourism Council. Todos los datos están referidos al año 2011.

España es, por tanto, el cuarto país del mundo en número de llegadas de turistas extranjeros, el segundo en ingresos y, entre ellos, el que cuenta con un mayor peso de este sector en la economía nacional, tanto en términos de Producto Interno Bruto (PIB) como de empleo.

En cuanto a la producción investigadora, hemos tomado la base de datos ISI Web of Science ${ }^{1}$, considerada en la actualidad como la principal plataforma de información científica. De hecho, la publicación en revistas incluidas en los índices del Institute for Scientific Information (ISI) suele ser la principal referencia a la hora de evaluar la actividad investigadora.

Sería lógico que aquellos países en los que el turismo tiene mayor peso a escala global fueran también países líderes en la producción de conocimiento científico para alimentar la industria turística. España es uno

1 Incluye: Science Citation Index Expanded (SCI-EXPANDED), Social Sciences Citation Index (SSCI), Arts \& Humanities Citation Index (A\&HCI), Conference Proceedings Citation Index-Science (CPCI-S) y Conference Proceedings Citation Index-Social Science \& Humanities (CPCI-SSH). 
de esos países y, por ello, nos proponemos conocer cuál es su posición en materia de investigación en turismo, en términos relativos respecto al resto de países líderes. Además, como ha quedado patente, es el país en cuya economía el turismo tiene un mayor peso.

El horizonte temporal del estudio abarca los años transcurridos del presente siglo: es decir, el período comprendido entre 2001 y 2012.

\section{Documentos sobre turismo}

La primera búsqueda fue realizada utilizando "Tourism" como tópico y los referidos países como "Address", para ubicar la procedencia de los documentos extraídos. El resultado fue el que se recoge en la Tabla A1 (ver Anexo), de la que destacamos lo siguiente:

- La producción documentaria en España es más elevada en relación con el número total de documentos contabilizados $(81 \%)$.

- Cuantitativamente ocupa el quinto lugar, detrás de Estados Unidos (que claramente lidera), China, Inglaterra y Australia.

- Llama la atención el muy rezagado lugar de Francia, primer país receptor de turismo internacional. Asimismo, la penetración de la lengua inglesa es la más baja entre todos los países punteros.

En cuanto a la evolución experimentada en los 12 años del presente siglo (Tabla A2, en Anexo), es claro que, en general, se ha producido un crecimiento muy notable de la producción científica. En el caso de España se ha multiplicado casi por diez, incluso más si tomamos el pico alcanzado en 2011. No obstante, el crecimiento más espectacular es el de China que, de mantenerse, pronto alcanzará una posición de liderazgo.

La investigación en estos años ha estado dominada por dos grandes temas o enfoques (Tabla A3, en Anexo): el de los estudios y ciencias medioambientales (predominante en casi todos los países, menos en China y Malasia), y el de la economía y los negocios. Tras ambos países, España es el país en el que la temática de economía y empresa ostenta un mayor peso en la producción científica sobre turismo.

\section{Artículos sobre turismo}

Nos concentraremos seguidamente en la parte más importante de la producción científica: los artículos.

En este apartado, y según la Tabla A5 (ver Anexo), la supremacía de Estados Unidos e Inglaterra se puede ver amenazada a mediano plazo por China. España ocupa el cuarto lugar por número de artículos ISI. Tomando las tres principales revistas de turismo por su factor de impacto en el la edición 2011 del Índice de Ciencias Sociales de JCR, Inglaterra y China son los países con un mayor peso de producción en esas tres revistas. En un segundo nivel se encuentran, con porcentajes muy semejantes, Estados Unidos, España y Turquía (Tabla A4, en Anexo).

Reino Unido (UK) no aparece como tal, por lo que hemos utilizado en su lugar Inglaterra (England). 
Aparte del espectacular crecimiento de China, solo los crecimientos de Malasia y Turquía son parangonables al de España, que ha multiplicado su producción de artículos por más de once (casi por catorce si tomamos la referencia de los artículos publicados en 2011).

La Tabla A6 (ver Anexo) lista las universidades españolas con mayor producción de artículos ISI en el período 2001-2012, con las de las Islas Baleares y Alicante claramente a la cabeza. Por comunidades autónomas, la distribución de los autores nacionales es la siguiente (Tabla 2):

\section{Tabla 2}

\begin{tabular}{lllll}
\hline Comunidad Autónoma & $\mathbf{N}^{\mathbf{0}}$ & $\mathbf{\%}$ & $\mathbf{R K}$ & Universidades con mayor contribución \\
\hline ANDALUCÍA & 123 & 16,2 & 2 & Granada (25), Málaga (24) y Sevilla (21) \\
ARAGÓN & 14 & 1,8 & 12 & Zaragoza (14) \\
ASTURIAS & 8 & 1,1 & $15-16-17$ & Oviedo (8) \\
BALEARES & 79 & 10,4 & 5 & Islas Baleares (79) \\
COMUNIDAD VALENCIANA & 111 & 14,6 & 3 & Alicante (61) y Valencia (22) \\
CANARIAS & 53 & 7,0 & 6 & Las Palmas Gran Canaria (32) y La Laguna (21) \\
CANTABRIA & 13 & 1,7 & $13-14$ & Cantabria (13) \\
CATALUÑA & 132 & 17,4 & 1 & Aut. Barcelona (34), Barcelona (30) y Girona (22) \\
CASTILLA - LA MANCHA & 20 & 2,6 & 9 & Castilla - La Mancha (20) \\
CASTILLA Y LEÓN & 24 & 3,2 & 8 & Valladolid (10) \\
EXTREMADURA & 8 & 1,1 & $15-16-17$ & Extremadura (8) \\
GALICIA & 33 & 4,3 & 7 & Santiago (15) y Vigo (15) \\
COMUNIDAD DE MADRID & 83 & 10,9 & 4 & Autónoma (26) y Complutense (26) \\
REGIÓN DE MURCIA & 13 & 1,7 & $13-14$ & Murcia (12) \\
COMUNIDAD F. DE NAVARRA & 18 & 2,4 & 11 & Navarra (14) \\
PAíS VASCO & 19 & 2,5 & 10 & País Vasco (16) \\
UNED & 8 & 1,1 & $15-16-17$ & Universidad Nacional de Educación a Distancia (8) \\
Total & 759 & 100 & - & - \\
\hline
\end{tabular}

Fuente: Elaboración propia con datos de ISI Web of Science.

Se observa una clara dicotomía entre las comunidades autónomas del Estado español, con un bloque de seis que concentran casi el $80 \%$ de los autores: Cataluña, Andalucía, Comunidad Valenciana, Madrid, Baleares y Canarias. En el resto la investigación en turismo se ha desarrollado poco.

En cuanto a las fuentes donde se han publicado los artículos, el Boletín de la Asociación de Geógrafos Españoles debe ser destacado, por tratarse del tercer título con más artículos de turismo publicados (Tabla A7, en Anexo), solo por detrás de Tourism Management y Tourism Economics.

Los temas ambientales/ecológicos, junto con tópicos de las ciencias sociales, han sido los más tratados (Tabla A8, en Anexo). La economía de la empresa y la geografía destacan a continuación, con la sociología y otros ya a bastante distancia. 
En el epígrafe siguiente nos centraremos en la categoría dominante en España: "hospitalidad, ocio, deporte y turismo", con prácticamente un tercio de los artículos, según expresa la Tabla A9 (ver Anexo). En este aspecto hay diferencias considerables entre países, con China-Malasia (con más de la mitad) y México en extremos opuestos.

\section{Artículos sobre hospitalidad, ocio, deporte y turismo}

Como se deriva de la Tabla A10 (ver Anexo), España es el país cuya producción de artículos publicados en revistas ISI ha crecido más en los últimos años dentro de este ámbito. Esta evolución le permite situarse en el quinto lugar por número total de artículos publicados en lo que llevamos de este siglo, tan solo por detrás de Estados Unidos, Australia, Inglaterra y China.

La Tabla A11 (ver Anexo) detalla las principales universidades españolas por su producción de artículos ISI en esta categoría, destacando nuevamente las de Islas Baleares y Alicante. Asimismo, la Tabla A13 (en Anexo) completa este panorama con las universidades líderes en cada uno de los países considerados.

Por comunidades autónomas la distribución de los autores nacionales es la que sigue (Tabla 3):

\section{Tabla 3}

\begin{tabular}{lllll}
\hline Comunidad Autónoma & $\mathbf{N}^{\mathbf{0}}$ & $\%$ & $\mathbf{R K}$ & Universidades con mayor contribución \\
\hline ANDALUCÍA & 35 & 13,8 & 4 & Málaga (12) \\
ARAGÓN & 4 & 1,6 & $11-12$ & Zaragoza (4) \\
ASTURIAS & 1 & 0,4 & 17 & Oviedo (1) \\
BALEARES & 47 & 18,6 & 1 & Baleares (47) \\
COMUNIDAD VALENCIANA & 43 & 17,0 & 2 & Alicante (26) \\
CANARIAS & 24 & 9,5 & 5 & Las Palmas de Gran Canaria (15) \\
CANTABRIA & 4 & 1,6 & $11-12$ & Cantabria (4) \\
CATALUÑA & 38 & 15,0 & 3 & Barcelona (11) y Girona (11) \\
CASTILLA - LA MANCHA & 3 & 1,2 & $13-14-15-16$ & Castilla -La Mancha (3) \\
CASTILLA Y LEÓN & 7 & 2,8 & 8 & Valladolid (4) \\
EXTREMADURA & 3 & 1,2 & $13-14-15-16$ & Extremadura (3) \\
GALICIA & 6 & 2,4 & $9-10$ & Vigo (4) \\
COMUNIDAD DE MADRID & 17 & 6,7 & 6 & Autónoma (7) \\
REGIÓN DE MURCIA & 3 & 1,2 & $13-14-15-16$ & Murcia (3) \\
COMUNIDAD F. DE NAVARRA & 9 & 3,6 & 7 & Navarra (6) \\
PAíS VASCO & 3 & 1,2 & $13-14-15-16$ & País Vasco (2) \\
UNED & 6 & 2,4 & $9-10$ & Universidad Nacional de Educación a Distancia (6) \\
Total & 253 & 100 & - & - \\
\hline
\end{tabular}

Fuente: Elaboración propia con datos de ISI Web of Knowledge. 
En esta categoría la concentración de la producción científica es aún mayor, con cinco comunidades claramente destacadas, en este orden: Baleares, Comunidad Valenciana, Cataluña, Andalucía y Canarias; obviamente las regiones turísticas por antonomasia. Obsérvese en la Tabla 4 el enorme impacto que el turismo tiene en las Islas Baleares (y también en Canarias).

Tabla 4

\begin{tabular}{lccccc}
\hline$\%$ & Baleares & Canarias & C. Valenc. & Andalucía & España \\
\hline PIB & 43,2 & 27,4 & 12,8 & 12,5 & 10,0 \\
EMPLEO & 30,3 & 30,2 & 12,6 & 11,9 & 7,3 \\
GASTO PÚBLICO & 11,2 & 9,1 & 5,4 & 2,9 & 1,2 \\
IMPUESTOS & 39,1 & 30,7 & 11,9 & 12,5 & - \\
\hline
\end{tabular}

Fuente: Exceltur. Estudio Impactur, Islas Baleares (2009).

En cuanto a las revistas en que esos artículos han sido publicados, Tourism Management, Tourism Economics, Annals of Tourism Research e International Journal of Tourism Research son las que ostentan la primacía en España, en ese orden. El listado de las diez fuentes más relevantes se recoge en la Tabla A12 (ver Anexo). La Tabla A14 del Anexo la complementa con la indicación de los títulos más recurrentes en el resto de países considerados.

\section{Las revistas ISI}

Mejorar el posicionamiento como país requiere, claramente, incrementar el número de artículos publicados en este tipo de revistas, que son las mejor consideradas en la comunidad científica. A tal efecto, recogemos:

1. Dentro de la categoría "Hospitality, Leisure, Sport \& Tourism” encontramos, en la edición 2011 del Índice de Ciencias Sociales JCR, un total de 36 revistas. Centrándonos en las que corresponden a turismo y hospitalidad, nos quedamos con las 16 siguientes (Tabla 5), ordenadas por factor de impacto. 
Tabla 5

\begin{tabular}{|c|c|c|c|c|c|c|c|}
\hline \multirow{2}{*}{ Título abreviado } & \multirow{2}{*}{ ISSN } & \multicolumn{6}{|c|}{ JCR Data } \\
\hline & & Total de citas & $\begin{array}{l}\text { Factor de } \\
\text { impacto }\end{array}$ & $\begin{array}{l}\text { Factor de } \\
\text { impacto (5 años) }\end{array}$ & $\begin{array}{l}\text { Índice de } \\
\text { inmediatez }\end{array}$ & Artículos & $\begin{array}{r}\text { Vida media } \\
\text { de citas }\end{array}$ \\
\hline ANN TOURISM RES & $0160-7383$ & 3646 & 3.259 & 3.356 & 0.183 & 71 & $>10.0$ \\
\hline TOURISM MANAGE & $0261-5177$ & 4391 & 2.597 & 3.701 & 0.401 & 152 & 6.5 \\
\hline J SUSTAIN TOUR & 0966-9582 & 954 & 1.929 & & 0.767 & 43 & 5.7 \\
\hline INT J HOSP MANAG & 0278-4319 & 1265 & 1.771 & & 0.061 & 114 & 5.2 \\
\hline J TRAVEL RES & $0047-2875$ & 2411 & 1.579 & & 0.098 & 51 & $>10.0$ \\
\hline J HOSP TOUR RES & $1096-3480$ & 462 & 0.943 & & 0.000 & 24 & 7.1 \\
\hline INT J CONTEMP HOSP M & $0959-6119$ & 906 & 0.929 & & 0.019 & 53 & 7.5 \\
\hline CORNELL HOSP Q & $1938-9655$ & 116 & 0.878 & 0.953 & 0.106 & 47 & 2.7 \\
\hline TOURISM GEOGR & $1461-6688$ & 328 & 0.840 & & 0.037 & 27 & 6.9 \\
\hline CURR ISSUES TOUR & $1368-3500$ & 297 & 0.836 & & 0.256 & 43 & 5.7 \\
\hline INT J TOUR RES & $1099-2340$ & 488 & 0.816 & & 0.075 & 40 & 6.9 \\
\hline SCAND J HOSP TOUR & $1502-2250$ & 174 & 0.630 & & 0.065 & 31 & 4.6 \\
\hline TOURISM ECON & $1354-8166$ & 606 & 0.579 & & 0.013 & 76 & 7.0 \\
\hline J HOSP LEIS SPORT TO & $1473-8376$ & 61 & 0.346 & & 0.000 & 29 & \\
\hline ASIA PAC J TOUR RES & $1094-1665$ & 140 & 0.300 & & 0.027 & 37 & 6.4 \\
\hline J TOUR CULT CHANGE & $1476-6825$ & 42 & 0.175 & & 0.000 & 22 & \\
\hline
\end{tabular}

Fuente: 2011 JCR Social Science Edition.

2. La información acerca de las características de las principales revistas es importante para que los potenciales interesados puedan elegir la más adecuada en función del perfil del trabajo que se pretende publicar.

Así, Svensson, Svaeri y Einarsen (2009), en un análisis de los trabajos empíricos publicados en las revistas que aparecen en la Tabla 6 en el período comprendido entre los años 2000 y 2007, nos muestra el claro predominio de los estudios cuantitativos (en especial del Journal of Hospitality Tourism Research), siendo Annals of Tourism Research la revista con mayor tendencia hacia los estudios cualitativos y hacia aquellos que combinan ambas metodologías (triangular). 
Tabla 6

\begin{tabular}{lcccl}
\hline \multirow{2}{*}{ Título } & \multicolumn{3}{c}{ Diseño de la investigación empírica } \\
\cline { 2 - 5 } & Cuantitativo & Cualitativo & Triangular & Total \\
\hline Annals of Tourism Research & $51,5 \%$ & $22,4 \%$ & $26,2 \%$ & 340 \\
Cornell Hotel and Restaurant Quarterly & $65,7 \%$ & $20,6 \%$ & $13,7 \%$ & 175 \\
International Journal of Hospitality Management & $68,4 \%$ & $(23,2 \%)$ & $(8,3 \%)$ & 228 \\
Journal of Hospitality Tourism Research & $90,2 \%$ & $4,3 \%$ & $5,5 \%$ & 164 \\
Journal of Travel Research & $74,9 \%$ & $7,0 \%$ & $18,1 \%$ & 299 \\
Tourism Management & $65,4 \%$ & $13,8 \%$ & $20,9 \%$ & 508 \\
Total & $67,1 \%$ & $15,3 \%$ & $17,6 \%$ & $1.714(100 \%)$ \\
\hline
\end{tabular}

Fuente: Svensson, Svaeri y Einarsen (2009).

También puede ser interesante conocer las procedencias geográficas de los datos de los trabajos publicados en estas revistas. Tal y como refleja la Tabla 7, es llamativa la escasa presencia de artículos de América del Sur, llegando a estar ausentes por completo en el Journal of Hospitality Tourism Research. Se trata de la zona del mundo menos investigada, lo que revela una gran oportunidad, fruto del vacío existente.

Tabla 7

\begin{tabular}{lcccccc}
\hline \multicolumn{1}{c}{ Título } & África & Asia & Australia & Europa & $\begin{array}{c}\text { América } \\
\text { del Norte }\end{array}$ & $\begin{array}{c}\text { América } \\
\text { del Sur }\end{array}$ \\
\cline { 1 - 2 } Annals of Tourism Research & $3,3 \%$ & $18,1 \%$ & $12,1 \%$ & $34,0 \%$ & $31,5 \%$ & $1,1 \%$ \\
Cornell Hotel and Restaurant Quarterly & $1,0 \%$ & $9,6 \%$ & $4,5 \%$ & $8,1 \%$ & $72,7 \%$ & $0,4 \%$ \\
International Journal of Hospitality Management & $1,2 \%$ & $22,7 \%$ & $8,5 \%$ & $30,0 \%$ & $36,4 \%$ & $1,2 \%$ \\
Journal of Hospitality Tourism Research & $0,6 \%$ & $17,6 \%$ & $8,5 \%$ & $8,5 \%$ & $64,8 \%$ & - \\
Journal of Travel Research & $2,9 \%$ & $31,1 \%$ & $14,1 \%$ & $33,5 \%$ & $17,8 \%$ & $0,6 \%$ \\
Tourism Management & $1,0 \%$ & $13,7 \%$ & $18,1 \%$ & $19,4 \%$ & $47,2 \%$ & $0,6 \%$ \\
Total & $2,0 \%$ & $22,0 \%$ & $12,8 \%$ & $27,1 \%$ & $41,2 \%$ & $1,2 \%$ \\
\hline
\end{tabular}

Fuente: Svensson, Svaeri y Einarsen (2009).

3. En la medida en que conectemos con los temas prioritarios que marcan la agenda de investigación en este campo científico, las posibilidades de tener cabida en este tipo de revistas top serán mayores.

Por ejemplo, Holmes, Jones, Lockwood, Miller, Scarles, Szivas y Tribe (2006) plantean cinco grandes temáticas: metodologías de investigación, educación en turismo, cultura y patrimonio, gestión de la hospitalidad (hotelería) y turismo sostenible. 
Brunelli, Macedo-Soares, Zouain y Borger (2010), por su parte, delimitan una serie de macrolíneas de investigación, a partir de una revisión de artículos publicados en el quinquenio 2005-2009 (entre paréntesis el porcentaje de artículos clasificados en cada categoría), las que se listan a continuación (Tabla 8).

Tabla 8

\begin{tabular}{|c|c|c|c|}
\hline ESTRATEGIA (14,2\%) & $\begin{array}{l}\text { - Ventaja competitiva. } \\
\text { - Competitividad. } \\
\text { - Alianzas estratégicas y redes. } \\
\text { - Análisis de los grupos de interés. } \\
\text { - Desempeño. }\end{array}$ & DIRECCIÓN $(8,2 \%)$ & $\begin{array}{l}\text { - Dirección. } \\
\text { - Recursos humanos. } \\
\text { - Gestión del turismo. } \\
\text { - Externalización. }\end{array}$ \\
\hline MÁRKETING (34,5\%) & $\begin{array}{l}\text { - Consumidor. } \\
\text { - Imagen/Marca. } \\
\text { - Análisis del mercado. } \\
\text { - Compras. } \\
\text { - Identidad/Percepción. }\end{array}$ & DESARROLLO $(12,3 \%)$ & $\begin{array}{l}\text { - Desarrollo local. } \\
\text { - Comunidades. } \\
\text { - Impactos económicos. } \\
\text { - Globalización. } \\
\text { - Exclusión social. }\end{array}$ \\
\hline SOSTENIBILIDAD (8,8\%) & $\begin{array}{l}\text { - Desarrollo sostenible. } \\
\text { - Turismo sostenible. } \\
\text { - Impactos del turismo. } \\
\text { - Medio ambiente. } \\
\text { - Responsabilidad social. }\end{array}$ & POLÍTICAS PÚBLICAS (2,5\%) & $\begin{array}{l}\text { - Gobernanza. } \\
\text { - Administración pública. } \\
\text { - Formulación de políticas. } \\
\text { - Política económica. }\end{array}$ \\
\hline NICHOS $(13,9 \%)$ & $\begin{array}{l}\text { - Patrimonio histórico y cultural. } \\
\text { - Turismo cultural. } \\
\text { - Turismo religioso. } \\
\text { - Turismo de aventura. } \\
\text { - Turismo senior. }\end{array}$ & INVESTIGACIÓN (5,6\%) & $\begin{array}{l}\text { - Epistemología. } \\
\text { - Metodologías. } \\
\text { - Taxonomías. } \\
\text { - Estudios críticos. } \\
\text { - Investigación interdisciplinaria. }\end{array}$ \\
\hline
\end{tabular}

Finalmente la OMT, en el Foro del Algarve de junio de 2011, también ha establecido una agenda de investigación centrada en tres grandes líneas: desarrollo y competitividad, cambio climático y sostenibilidad, gobernanza y política turística.

\section{Conclusiones}

El presente trabajo comenzó con unas interrogantes a las que debemos dar respuesta.

Si comparamos la posición de los países según el número de llegadas internacionales de turistas con la producción de artículos ISI sobre turismo en general (Tabla 9), España se encuentra al mismo nivel, verificándose una correspondencia entre ambas variables (cuarto lugar en ambos casos). Por otra parte, países como Estados Unidos y Reino Unido (las dos principales potencias investigadoras) cuentan con una posición en términos de producción científica mejor que la que ocupan en el referido ránking de destinos turísticos; situación opuesta a la de Francia, cuya posición en cuanto a producción científica está muy lejos (décimo tercer lugar) del liderazgo que ostenta como destino receptor de turismo internacional. 
Si efectuamos la comparación con los ingresos generados por el flujo de turistas internacionales, Estados Unidos es el líder aventajado desde ambos puntos de vista. España, siendo el segundo país generador de ingresos, es el cuarto en producción científica, aunque Francia, que le sigue, está bastante peor posicionada, en los términos ya referidos.

Tabla 9

\begin{tabular}{clc}
\hline Posición en el ránking & Millones de turistas internacionales (2011) & $\begin{array}{c}\mathbf{N}^{0} \text { de artículos publicados bajo el tópico } \\
\text { "Tourism" (2001-2012) }\end{array}$ \\
\hline 1 & Francia & EE.UU. \\
2 & EE.UU. & Inglaterra \\
3 & China & Australia \\
4 & España & España \\
5 & Italia & China \\
6 & Turquía & Canadá \\
7 & Reino Unido & Italia \\
8 & Alemania & Taiwán \\
9 & Malasia & Alemania \\
10 & México & Turquía \\
\hline
\end{tabular}

Fuente: Elaboración propia.

No obstante:

- La producción de artículos científicos de España en la categoría "Hospitality, Leisure, Sport \& Tourism" nos sitúa un puesto más abajo (quinto), habiendo sido desbancados por China, cuyo crecimiento hace presagiar que pronto nos superará en producción total en materia de turismo, incluso amenazando a mediano plazo la supremacía anglosajona ya referida.

- La producción de artículos ISI sobre turismo se ha multiplicado por seis en la última década (2003-2012), pasando en la categoría "Hospitality, Leisure, Sport \& Tourism" a multiplicarse casi por diez en ese mismo período. Esto da idea del fuerte crecimiento experimentado y de la creciente (además de rápida) inserción internacional de la investigación española en turismo.

- Por comunidades autónomas sigue existiendo una clara correspondencia entre las regiones turísticas por excelencia y el peso de las publicaciones científicas a escala internacional: el arco mediterráneo, Andalucía y Canarias son las más destacadas. Las universidades de las Islas Baleares y de Alicante han sido las más activas en lo que va del siglo. 


\section{Referencias}

Aguiló, E. \& Vich, G. A. (1996). La investigación en el ámbito de la política turística. Estudios Turísticos, 129, 23-36.

Albacete-Sáez, C. A. \& Fuentes-Fuentes, M. M. (2010). Difusión de la investigación española sobre turismo en revistas internacionales. Análisis Turístico, 9, 14-29.

Antón, S., López i Palomeque, F., Marchena, M. \& Vera, J. F. (1996). La investigación turística en España. Estudios Turísticos, 129, 165-208.

Ballantyne, R., Packer, J. \& Axelsen, M. (2009). Trends in tourism research. Annals of Tourism Research, 36(1), $149-152$.

Bigné, J. E. (1996). Turismo y Marketing en España: Análisis del estado de la cuestión y perspectivas de futuro. Estudios Turísticos, 129, 105-128.

Bote, V. (1996). La investigación en España sobre el turismo y desarrollo económico. Estudios Turísticos, 129, 9-22.

Brunelli, M. Q., Macedo-Soares, T. D. L., Zouain, D. M. \& Borger, A. P. (2010). Scientific research in tourism: review of the literature from 2005 to 2009. Revista de Administração Pública, 44(5), 1225-1240.

Bueno, E. J. (1996). La investigación sobre las empresas turísticas en España: el estado del arte. Estudios Turísticos, 129, 61-80.

Dann, G. (2011). Anglophone Hegemony in Tourism Studies Today. Enlightening Tourism. A Pathmaking Journal, 1(1), 1-30. Recuperado de http://www.uhu.es/publicaciones/ojs/index.php/et/article/view/1017

De la Calle, M. \& García, M. (2004). La investigación geográfica del turismo en España. Anales de Geografía de la Universidad Complutense, 24, 257-277.

Esteban, A. (2000). La investigación turística en la universidad española. Estudios Turísticos, 144-145, 155-180.

Esteban, A. \& Reinares, E. M. (1996). La investigación de la demanda turística en España: recopilación y análisis. Estudios Turísticos, 129, 81-104.

Etzkowitz, H. (2008). The Triple Helix: University-Industry-Government Innovation In Action. London: Routledge.

Figuerola, M. (2000). Los trabajos de investigación de la economía del turismo en el Instituto de Estudios Turísticos: 1974-1993. Estudios Turísticos, 144-145, 17-46.

Holmes, K., Jones, P., Lockwood, A., Miller, G., Scarles, C., Szivas, E. \& Tribe, J. (2006). An eclectic agenda for Tourism and Hospitality Research. Tourism and Hospitality Research, 7(1), 76-82.

Jones, P. A. \& Phillips, D. (2003). What use is research anyway? Industry and academie's differing views. International Journal of Contemporary Hospitality Management, 15(5), 290-293.

Ortega, E. \& Rodríguez, B. (2004). La investigación turística a través de tesis doctorales: Un análisis comparativo entre España y Francia. Estudios Turísticos, 159, 5-28.

Sánchez, M. \& Marín, M. B. (2003). La investigación en turismo y economía de la empresa publicada en revistas especializadas españolas: 1996-2001. Papers de Turisme, 33, 6-39.

Svensson, G., Svaeri, S. \& Einarsen, K. (2009). Empirical characteristics' of scholarly journals in hospitality and tourism research: An assessment. International Journal of Hospitality Management, 28(3), 479-483.

Vera, J. F. \& Ivars, J. A. (2001). La formación y la investigación turística en España: una visión de síntesis. Papers de Turisme, 29, 627.

Vargas-Sanchez, A. (2011a). Tourism Research in Spain: The State of the Art. Enlightening Tourism. A Pathmaking Journal, 1(1), 93110. Recuperado de http://www.uhu.es/publicaciones/ojs/index.php/et/article/view/1019

Vargas-Sánchez, A. (2011). ¿Los principales destinos son también las principales potencias en la investigación en turismo? Estudios Turísticos, 188, 91-111. 


\section{ANEXO}

(Fuente: ISI Web of Science, 2001-2012)

Tabla A1

Distribución por países de los tipos de documentos publicados bajo el tópico "Tourism" (2001-2012)

\begin{tabular}{lrrrrrrrrrr}
\hline Tipo dedocumento & FRANCIA & EEUU & CHINA & ESPAÑA & ITALIA & TURQUíA & INGLATERRA & ALEMANIA & MALASIA MÉXICO \\
\hline $\mathrm{N}^{\circ}$ total de docs $\left(\mathrm{Rk}^{*}\right)$ & $329\left(12^{\circ}\right)$ & $3,002\left(1^{\circ}\right)$ & $2,355\left(2^{\circ}\right)$ & $879\left(5^{\circ}\right)$ & $488\left(7^{\circ}\right)$ & $402\left(10^{\circ}\right)$ & $1,421\left(3^{\circ}\right)$ & $450\left(8^{\circ}\right)$ & $120\left(31^{\circ}\right)$ & $113\left(32^{\circ}\right)$ \\
$\mathrm{N}^{0}$ de artículos $\left(\mathrm{R}^{* *}\right)$ & $231\left(13^{\circ}\right)$ & $2,150\left(1^{\circ}\right)$ & $588\left(5^{\circ}\right)$ & $715\left(4^{\circ}\right)$ & $339\left(7^{\circ}\right)$ & $294\left(10^{\circ}\right)$ & $1,034\left(2^{\circ}\right)$ & $321\left(9^{\circ}\right)$ & $64\left(35^{\circ}\right)$ & $84\left(32^{\circ}\right)$ \\
$\%$ artículos & 70.213 & 71.619 & 24.968 & 81.342 & 69.467 & 73.134 & 72.766 & 71.333 & 53.333 & 74.336 \\
\hline
\end{tabular}

*Australia es $4^{\circ}$, Canadá $6^{\circ}$, Taiwán $9^{\circ}$, Perú $84^{\circ}$ (11 documentos).

${ }^{*}$ Australia es $3^{\circ}$, Canadá $6^{\circ}$, Taiwán $8^{\circ}$, Perú $81^{\circ}(9$ artículos $)$.

Tabla A2

Evolución temporal del número de documentos publicados bajo el tópico "Tourism"

\begin{tabular}{|c|c|c|c|c|c|c|c|c|c|c|}
\hline Tipo dedocumento & FRANCIA & EEUU & CHINA & ESPAÑA & ITALIA & TURQUÍA I & INGLATERRA & ALEMANIA & MALASIA & MÉXICO \\
\hline 2001 & 9 & 112 & 9 & 14 & 17 & 5 & 63 & 17 & 1 & 4 \\
\hline 2002 & 26 & 150 & 18 & 16 & 14 & 4 & 73 & 28 & 0 & 6 \\
\hline 2003 & 6 & 141 & 26 & 23 & 11 & 11 & 67 & 20 & 1 & 4 \\
\hline 2004 & 13 & 164 & 44 & 22 & 21 & 14 & 63 & 22 & 1 & 9 \\
\hline 2005 & 16 & 163 & 42 & 36 & 22 & 22 & 81 & 23 & 2 & 4 \\
\hline 2006 & 24 & 214 & 79 & 55 & 31 & 22 & 86 & 22 & 2 & 9 \\
\hline 2007 & 24 & 231 & 141 & 73 & 31 & 33 & 95 & 25 & 2 & 5 \\
\hline 2008 & 31 & 316 & 327 & 105 & 41 & 33 & 138 & 50 & 11 & 19 \\
\hline 2009 & 53 & 339 & 379 & 110 & 61 & 54 & 154 & 42 & 8 & 12 \\
\hline 2010 & 57 & 392 & 444 & 123 & 66 & 65 & 189 & 63 & 26 & 11 \\
\hline 2011 & 32 & 401 & 517 & 164 & 96 & 88 & 206 & 74 & 39 & 12 \\
\hline 2012 & 38 & 379 & 329 & 138 & 77 & 51 & 206 & 64 & 27 & 18 \\
\hline Total & 329 & 3,002 & 2,355 & 879 & 488 & 402 & 1,421 & 450 & 120 & 113 \\
\hline$\%$ en inglés & 79.635 & 99.567 & 94.268 & 86.576 & 98.770 & 93.532 & 99.859 & 87.333 & 99.167 & 79.646 \\
\hline
\end{tabular}

Tabla A3

Peso de las principales áreas temáticas en la producción científica de cada país bajo el tópico “Tourism” (2001-2012)

\begin{tabular}{|c|c|c|c|c|c|c|c|c|c|c|}
\hline $\begin{array}{l}\text { Principales áreas } \\
\text { temáticas (\%) }\end{array}$ & FRANCIA & EEUU & CHINA & ESPAÑA & ITALIA & TURQUÍA & INGLATERRA & ALEMANIA & MALASIA & MÉXICO \\
\hline $\begin{array}{l}\text { ENVIRONMENTAL } \\
\text { SCIENCES / ECOLOGY }\end{array}$ & 30.395 & 23.185 & 27.304 & 30.830 & 31.967 & 35.821 & 26.319 & 24.889 & 16.667 & 32.743 \\
\hline BUSINESS & & & & & & & & & & \\
\hline ECONOMICS & 14.286 & 16.589 & 39.830 & 26.507 & 18.238 & 22.886 & 21.816 & 10.444 & 28.333 & 8.850 \\
\hline
\end{tabular}


Tabla A4

Peso de la producción científica de cada país (artículos) bajo el tópico "Tourism" (2001-2012) en las tres principales revistas de turismo por factor de impacto en JCR Social Science2011

\begin{tabular}{|c|c|c|c|c|c|c|c|c|c|c|}
\hline Principales revistas (\%) & FRANCIA & EEUU & CHINA & ESPAÑA & ITALIA & TURQUÍA & INGLATERRA & ALEMANIA & MALASIA & MÉXICO \\
\hline ANNALS OF & & & & & & & & & & \\
\hline $\begin{array}{l}\text { TOURISM RESEARCH } \\
\text { TOURISM }\end{array}$ & 1.732 & 6.512 & 4.932 & 4.615 & 2.065 & 3.741 & 11.509 & 0.935 & - & 1.190 \\
\hline MANAGEMENT & 3.030 & 8.233 & 14.116 & 11.329 & 7.080 & 11.565 & 9.188 & 2.804 & 6.250 & - \\
\hline $\begin{array}{l}\text { JOURNAL OF } \\
\text { SUSTAINABLE TOURISM }\end{array}$ & - & 2.093 & 2.211 & 0.559 & - & 1.020 & 1.934 & 1.246 & 4.688 & - \\
\hline Total & 4.762 & $\begin{array}{ll}16.838 \\
\end{array}$ & 21.259 & 16.503 & 9.145 & 16.326 & 22.631 & 4.985 & 10.938 & 1.190 \\
\hline
\end{tabular}

Tabla A5

Evolución temporal del número de artículos publicados bajo el tópico "Tourism" en cada país

\begin{tabular}{cllllllllll}
\hline $\mathrm{N}^{\circ}$ de artículos & FRANCIA & EEUU & CHINA & ESPAÑA & ITALIA & TURQUÍA & INGLATERRA & ALEMANIA & MALASIA & MÉXICO \\
\hline 2001 & 7 & 72 & 6 & 10 & 13 & 4 & 41 & 14 & 1 & 4 \\
2002 & 12 & 93 & 14 & 12 & 11 & 4 & 46 & 16 & 0 & 4 \\
2003 & 0 & 96 & 12 & 19 & 7 & 9 & 49 & 15 & 0 & 1 \\
2004 & 7 & 104 & 20 & 15 & 9 & 10 & 36 & 11 & 1 & 5 \\
2005 & 9 & 107 & 18 & 29 & 14 & 16 & 57 & 13 & 1 & 1 \\
2006 & 19 & 127 & 26 & 44 & 18 & 19 & 56 & 9 & 0 & 5 \\
2007 & 15 & 166 & 25 & 55 & 20 & 24 & 67 & 19 & 2 & 2 \\
2008 & 21 & 216 & 64 & 92 & 27 & 23 & 101 & 41 & 5 & 16 \\
2009 & 41 & 252 & 69 & 91 & 41 & 40 & 110 & 29 & 5 & 9 \\
2010 & 41 & 300 & 100 & 92 & 47 & 54 & 146 & 45 & 13 & 10 \\
2011 & 26 & 310 & 129 & 138 & 65 & 51 & 160 & 57 & 18 & 10 \\
2012 & 33 & 307 & 105 & 118 & 67 & 40 & 165 & 52 & 18 & 17 \\
Total & 231 & 2,150 & 588 & 715 & 339 & 294 & 1.034 & 321 & 64 & 84 \\
$\%$ en inglés & 78.355 & 99.488 & 100 & 86.014 & 98.525 & 91.156 & 99.807 & 84.424 & 98.438 & 77.381 \\
\hline
\end{tabular}

Tabla A6

Principales instituciones responsables de la producción científica española (artículos) bajo el tópico "Tourism" (2001-2012)

\begin{tabular}{llcc}
\hline Rk & Principales instituciones & N $^{\circ}$ de artículos & $\%$ \\
\hline 1 & UNIV ISLAS BALEARES & 72 & 10.070 \\
2 & UNIV ALICANTE & 61 & 8.531 \\
3 & UNIV BARCELONA & 29 & 4.056 \\
4 & UNIV AUTÓNOMA BARCELONA & 28 & 3.916 \\
5 & UNIV LAS PALMAS DE GRAN CANARIA & 27 & 3.776 \\
$6-7$ & CSIC & 25 & 3.497 \\
& UNIV GRANADA & 25 & 3.497 \\
8 & UNIV MÁLAGA & 24 & 3.357 \\
9 & UNIV AUTÓNOMA MADRID & 23 & 3.217 \\
$10-11$ & UNIV GIRONA - UNIV VALENCIA & 22 & 3.077 \\
\hline
\end{tabular}


Tabla A7

Principales revistas que recogen la producción científica española (artículos) bajo el tópico "Tourism" (2001-2012)

\begin{tabular}{llcc}
\hline Rk & Principales revistas & N $^{0}$ de artículos & $\%$ \\
\hline 1 & TOURISM MANAGEMENT & 81 & 11.329 \\
2 & TOURISM ECONOMICS & 52 & 7.273 \\
3 & BOLETIN DE LA ASOCIACION DE GEOGRAFOS ESPANOLES & 39 & 5.455 \\
4 & ANNALS OF TOURISM RESEARCH & 33 & 4.615 \\
5 & SERVICE INDUSTRIES JOURNAL & 18 & 2.517 \\
$6-7$ & INTERNATIONAL JOURNAL OF TOURISM RESEARCH & 16 & 2.238 \\
& SCRIPTANOVAREVISTAELECTRONICADE GEOGRAFIAY CIENCIAS SOCIALES & 16 & 2.238 \\
8 & JOURNAL OF COASTAL RESEARCH & 12 & 1.678 \\
9 & OCEAN COASTAL MANAGEMENT & 11 & 1.538 \\
10 & JOURNAL OF TRAVEL RESEARCH & 9 & 1.259 \\
\hline
\end{tabular}

Tabla A8

Principales áreas temáticas de la producción científica española (artículos) bajo el tópico "Tourism" (2001-2012)

\begin{tabular}{llcc}
\hline Rk & Principales áreas temática & $\mathrm{N}^{0}$ de artículos & $\%$ \\
\hline 1 & SOCIAL SCIENCES OTHER TOPICS & 238 & 33.287 \\
2 & ENVIRONMENTAL SCIENCES ECOLOGY & 222 & 31.049 \\
3 & BUSINESS ECONOMICS & 197 & 27.552 \\
4 & GEOGRAPHY & 81 & 11.329 \\
5 & SOCIOLOGY & 42 & 5.874 \\
6 & ENGINEERING & 37 & 5.175 \\
7 & COMPUTER SCIENCE & 32 & 4.476 \\
8 & WATER RESOURCES & 30 & 4.196 \\
$9-10$ & GEOLOGY & 25 & 3.497 \\
& URBAN STUDIES & 25 & 3.497 \\
\hline
\end{tabular}

Tabla A9

Principales categorías de la producción científica española (artículos) bajo el tópico "Tourism" (2001-2012)

\begin{tabular}{llcc}
\hline Rk & Principales categorías científicas & No de artículos $^{\circ}$ & \% \\
\hline 1 & HOSPITALITY LEISURE SPORT TOURISM & 233 & 32.587 \\
$2-3$ & ENVIRONMENTAL STUDIES & 133 & 18.601 \\
& MANAGEMENT & 133 & 18.601 \\
4 & ENVIRONMENTAL SCIENCES & 96 & 13.427 \\
5 & GEOGRAPHY & 81 & 11.329 \\
6 & ECONOMICS & 47 & 6.573 \\
7 & SOCIOLOGY & 42 & 5.874 \\
8 & ECOLOGY & 38 & 5.315 \\
9 & BUSINESS & 36 & 5.035 \\
10 & WATER RESOURCES & 30 & 4.196 \\
\hline
\end{tabular}


Tabla A10

Evolución temporal del número de artículos publicados en la categoría "Hospitality, Leisure, Sport \&Tourism" en cada país.

\begin{tabular}{|c|c|c|c|c|c|c|c|c|c|c|}
\hline $\mathrm{N}^{\circ}$ de artículos & FRANCIA & EEUU & CHINA & ESPAÑA & ITALIA & TURQUÍA & INGLATERRA & ALEMANIA & MALASIA & MÉXICO \\
\hline 2001 & 0 & 17 & 4 & 1 & 2 & 3 & 17 & 0 & 0 & 0 \\
\hline 2002 & 0 & 31 & 3 & 3 & 1 & 2 & 13 & 1 & 0 & 0 \\
\hline 2003 & 0 & 20 & 2 & 5 & 1 & 3 & 19 & 1 & 0 & 0 \\
\hline 2004 & 1 & 28 & 7 & 6 & 0 & 3 & 11 & 0 & 0 & 1 \\
\hline 2005 & 1 & 29 & 5 & 8 & 0 & 6 & 17 & 0 & 0 & 0 \\
\hline 2006 & 0 & 34 & 9 & 17 & 3 & 5 & 16 & 1 & 0 & 0 \\
\hline 2007 & 2 & 47 & 12 & 19 & 0 & 9 & 27 & 2 & 0 & 0 \\
\hline 2008 & 2 & 109 & 37 & 29 & 7 & 8 & 54 & 2 & 2 & 0 \\
\hline 2009 & 4 & 99 & 45 & 28 & 9 & 5 & 46 & 1 & 4 & 0 \\
\hline 2010 & 7 & 119 & 64 & 33 & 14 & 15 & 63 & 6 & 8 & 2 \\
\hline 2011 & 7 & 135 & 75 & 36 & 18 & 12 & 57 & 12 & 10 & 0 \\
\hline 2012 & 10 & 129 & 56 & 48 & 22 & 11 & 83 & 13 & 10 & 0 \\
\hline TOTAL & 34 & 797 & 319 & 233 & 77 & 82 & 423 & 39 & 34 & 3 \\
\hline Ránking * & $22^{\circ}$ & $1^{\circ}$ & $4^{\circ}$ & $5^{\circ}$ & $13^{\circ}$ & $12^{\circ}$ & $3^{\circ}$ & $20^{\circ}$ & $23^{\circ}$ & $55^{\circ}$ \\
\hline$\%$ en inglés & 100 & 100 & 100 & 100 & 100 & 100 & 100 & 100 & 100 & 100 \\
\hline$\% \mathrm{~s} /$ total turismo & 14.719 & 37.070 & 54.252 & 32.587 & 22.714 & 27.891 & 40.909 & 12.150 & 53.125 & 3.571 \\
\hline
\end{tabular}

${ }^{*} 2^{\circ}$ es Australia.

Tabla A11

Principales instituciones responsables de la producción científica española (artículos) en la categoría "Hospitality, Leisure, Sport \&Tourism" (2001-2012)

\begin{tabular}{|c|c|c|c|}
\hline $\mathrm{Rk}$ & Principales instituciones & $\mathrm{N}^{0}$ de artículos & $\%$ \\
\hline 1 & UNIV ISLAS BALEARES & 46 & 19.742 \\
\hline 2 & UNIV ALICANTE & 26 & 11.159 \\
\hline \multirow[t]{2}{*}{$3-4$} & UNIV LAS PALMAS GRAN CANARIA & 12 & 5.150 \\
\hline & UNIV MÁLAGA & 12 & 5.150 \\
\hline \multirow[t]{2}{*}{$5-6$} & UNIV BARCELONA & 11 & 4.721 \\
\hline & UNIV GIRONA & 11 & 4.721 \\
\hline 7 & UNIV LA LAGUNA & 9 & 3.863 \\
\hline \multirow[t]{2}{*}{ 8-9 } & UNIV AUTÓNOMA MADRID & 7 & 3.004 \\
\hline & UNIV VALENCIA & 7 & 3.004 \\
\hline $10-11$ & UNIV NAVARRA - UNIV SEVILLA & 6 & 2.575 \\
\hline
\end{tabular}


Tabla A12

Principales revistas que recogen la producción cientifica española (artículos) en la categoría "Hospitality, Leisure, Sport \&Tourism" (2001-2012)

\begin{tabular}{llcc}
\hline Rk & Principales revistas & No de artículos & $\%$ \\
\hline 1 & TOURISM MANAGEMENT & 81 & 34.764 \\
2 & TOURISM ECONOMICS & 52 & 22.318 \\
3 & ANNALS OF TOURISM RESEARCH & 33 & 14.163 \\
4 & INTERNATIONAL JOURNAL OF TOURISM RESEARCH & 16 & 6.867 \\
5 & JOURNAL OF TRAVEL RESEARCH & 9 & 3.863 \\
6 & INTERNATIONAL JOURNAL OF HOSPITALITY MANAGEMENT & 7 & 3.004 \\
7 & JOURNAL OF TRAVEL TOURISM MARKETING & 6 & 2.575 \\
$8-9$ & CURRENT ISSUES IN TOURISM & 5 & 2.146 \\
& INTERNATIONAL JOURNAL OF CONTEMPORARY HOSPITALITY MANAGEMENT & 5 & 2.146 \\
10 & CORNELL HOSPITALITY QUARTERLY & 4 & 1.717 \\
\hline
\end{tabular}

Tabla A13

Principales instituciones de cada país responsables de la producción científica (artículos) en la categoría "Hospitality, Leisure, Sport \& Tourism" (2001-2012)

\begin{tabular}{|c|c|c|c|c|c|c|c|c|c|c|}
\hline Rk & FRANCIA & EEUU & CHINA & ESPAÑA & ITALIA & TURQUI'A & INGLATERRA & ALEMANIA & MALASIA & MÉXICO \\
\hline 1 & PERPIGNAN & $\begin{array}{l}\text { TEXAS } \\
\text { A\&M }\end{array}$ & $\begin{array}{c}\text { HONG KONG } \\
\text { POLYTECH }\end{array}$ & $\begin{array}{c}\text { ISLAS } \\
\text { BALEARES }\end{array}$ & BOLZANO & MUGLA & SURREY & MUNICH & MALAYA & $\begin{array}{l}\text { AUTONOMOUS } \\
\text { UNIV OF THE } \\
\text { STATE OF MEXICO }\end{array}$ \\
\hline 2 & SAVOIE & PURDUE & $\begin{array}{l}\text { SUN YAT } \\
\text { SEN }\end{array}$ & ALICANTE & BOLOGNA & $\begin{array}{c}\text { ADNAN } \\
\text { MENDERES } \\
- \\
\text { MUSTAFA } \\
\text { KEMAL }\end{array}$ & BOURNEMOUTH & $\begin{array}{c}\text { BREMERHAVEN } \\
\text { UNIV APPL SCI } \\
- \\
\text { JOHANNES } \\
\text { GUTENBERG } \\
\text { UNIV MAINZ }\end{array}$ & $\begin{array}{c}\text { SAINS } \\
\text { MALAYSIA }\end{array}$ & COLIMA \\
\hline 3 & $\begin{array}{c}\text { LILLE } 1 \\
\text { MONTPELLIER } \\
1\end{array}$ & $\begin{array}{l}\text { ARIZONA } \\
\text { STATE }\end{array}$ & HONG KONG & $\begin{array}{l}\text { LAS PALMAS } \\
\text { DE GRAN } \\
\text { CANARIA } \\
- \\
\text { MÁLAGA }\end{array}$ & SASSARI & BOGAZICI & NOTTINGHAM & JENA & NOTTINGHAM & \\
\hline
\end{tabular}

Tabla A14

Principales revistas que recogen la producción científica de cada país (artículos) en la categoría "Hospitality, Leisure, Sport \& Tourism" (2001-2012)



